\title{
Brazilian Researchers with Scientifically Established Careers in Institutions Outside Brazil
}

The Journal of the Brazilian Chemical Society (JBCS) has been publishing special thematic issues with the strategy of disseminating high quality scientific work that interests the Chemistry community worldwide with consequent international achievement. This issue (Brazilian Researchers Abroad) is devoted to publishing papers from Brazilian Researchers whose careers are scientifically established in institutions outside Brazil. We believe this is a nice opportunity for both, the invited contributors who would have their work recognized by a Brazilian journal, and the JBCS that would have the mission to give visibility to their papers.

Bearing in mind this idea, the editors of JBCS have invited Brazilian authors from different universities worldwide, and in the end, eight of them contributed with papers that are now being published in this issue. We were expecting more contributions, however, some of the authors, even agreeing to send their papers, did not submit them. Perhaps, one of the reasons could be to the fact that the JBCS is not among the top journals of chemistry in the world, also a journal covering all the areas of chemistry may not attract specialists from some areas of chemistry. We would certainly like to have a higher number of submissions; however, this does not diminish our enthusiasm with this initiative. All the papers were submitted to the peer reviewing system of JBCS, and in the end, eight papers are published, 06 articles and 02 accounts. These papers are highlighted with a logo specially created for this number, being similar to the cover image. The papers appear as the 08 first; however, as in a regular issue we have around 22 papers, we have decided to add fourteen others from the forthcoming papers. The last papers were chosen by the editors considering related areas of the ones published by the Brazilian Researchers from abroad.

We are very much thankful to the authors Adelia J. A. Aquino (Tianjin University, Texas Tech University, University of Natural Resources and Life Sciences Vienna), Alessandra S. Eustáquio (University of Illinois), Emerson F. Queiroz (University of Geneva), Roberto Aquino (Imperial College London), Rodrigo B. Andrade (Temple University), George L. Donati (Wake Forest University), João B. P. Soares (University of Alberta) and Leonardo S. Santos (University of Talca), who have contributed with their papers. We are very glad about their success worldwide and wish them to keep doing good chemistry.

Paulo Cezar Vieira Editor-in-Chief of the Journal of the Brazilian Chemical Society Universidade de São Paulo (USP), Ribeirão Preto-SP, Brazil

Roberto M. Torresi Editor of the Journal of the Brazilian

Chemical Society Universidade de São Paulo (USP), São Paulo-SP, Brazil 